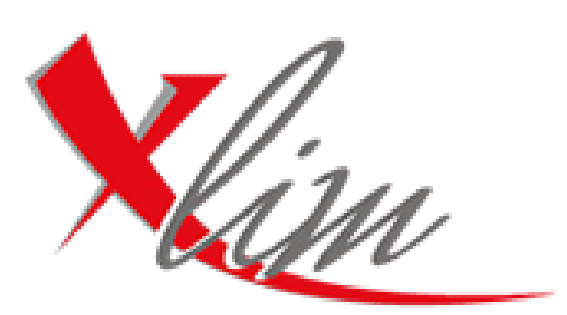

institut de recherche

\title{
High-Efficiency Harmonically Terminated Diode and Transistor Rectifiers
}

\author{
M. Roberg, T. Reveyrand, I. Ramos, E.A. Falkenstein, Z. Popović
}

\section{Published in}

IEEE Transactions on Microwave Theory and Techniques, Vol. 60, No 12, pp. 4043-4052, December 2012.

Crossref DOI: 10.1109/TMTT.2012.2222919

http://ieeexplore.ieee.org/xpl/articleDetails.jsp?arnumber=6353614

(C) 2012 IEEE Personal use of this material is permitted. However, permission to reprint/republish or redistribute this material for advertising or promotional purposes or for creating new collective works for resale or redistribution to servers or lists, or to reuse any copyrighted component of this work in other works must be obtained from the IEEE. 


\title{
High-Efficiency Harmonically Terminated Diode and Transistor Rectifiers
}

\author{
Michael Roberg, Student Member, IEEE, Tibault Reveyrand, Member, IEEE, Ignacio Ramos, Student Member, IEEE,
} Erez Avigdor Falkenstein, Student Member, IEEE, and Zoya Popović, Fellow, IEEE

\begin{abstract}
This paper presents a theoretical analysis of harmonically terminated high-efficiency power rectifiers and experimental validation on a class- $C$ single Schottky-diode rectifier and a class- $\mathrm{F}^{-1} \mathrm{GaN}$ transistor rectifier. The theory is based on a Fourier analysis of current and voltage waveforms, which arise across the rectifying element when different harmonic terminations are presented at its terminals. An analogy to harmonically terminated power amplifier (PA) theory is discussed. From the analysis, one can obtain an optimal value for the dc load given the RF circuit design. An upper limit on rectifier efficiency is derived for each case as a function of the device on-resistance. Measured results from fundamental frequency source-pull measurement of a Schottky diode rectifier with short-circuit terminations at the second and third harmonics are presented. A maximal device rectification efficiency of $72.8 \%$ at $2.45 \mathrm{GHz}$ matches the theoretical prediction. A 2.14-GHz GaN HEMT rectifier is designed based on a class- $F^{-1}$ PA. The gate of the transistor is terminated in an optimal impedance for self-synchronous rectification. Measurements of conversion efficiency and output de voltage for varying gate RF impedance, dc load, and gate bias are shown with varying input RF power at the drain. The rectifier demonstrates an efficiency of $85 \%$ for a $10-W$ input $R F$ power at the transistor drain with a dc voltage of $30 \mathrm{~V}$ across a $98-\Omega$ resistor.
\end{abstract}

Index Terms-Harmonic terminations, high-efficiency power amplifiers (PAs), load-pull, microwave rectifiers, nonlinear analysis, time-domain measurements.

\section{INTRODUCTION}

$\mathbf{T}$ HE FIRST RF rectifiers were demonstrated in experiments and patents in the 1890 s by Nikola Tesla in wireless power transmission for lighting applications and the method of obtaining direct from alternating current [1]. The

Manuscript received July 09, 2012; revised September 23, 2012; accepted September 25, 2012. Date of publication November 15, 2012; date of current version December 13, 2012. This work was supported in part by the Office of Naval Research (ONR) under the Defense Advanced Research Projects Agency (DARPA) Microscale Power Conversion (MPC) Program under Grant N00014-11-1-0931, and in part by the Advanced Research Projects Agency-Energy (ARPA-E), U.S. Department of Energy under Award Number DE-AR0000216. This paper is an expanded paper from the IEEE MTT-S International Microwave Symposium, Montreal, QC, Canada, June 17-22, 2012.

M. Roberg is with TriQuint Semiconductor, Richardson, TX 75080 USA (e-mail: michael.roberg@tqs.com).

T. Reveyrand is with the XLIM Laboratory, UMR 7252, University of Limoges, 87060 Limoges, France (e-mail: tibault.reveyrand@xlim.fr).

I. Ramos and Z. Popović are with the Department of Electrical, Computer and Energy Engineering, University of Colorado at Boulder, Boulder, $\mathrm{CO}$ 80309-0425 USA (e-mail: ignacio.ramos@colorado.edu; zoya.popovic@colorado.edu).

E. A. Falkenstein is with Qualcomm Inc., Boulder, CO 80301 USA (e-mail: erez.falkenstein@gmail.com).

Color versions of one or more of the figures in this paper are available online at http://ieeexplore.ieee.org.

Digital Object Identifier 10.1109/TMTT.2012.2222919 main application of microwave power rectifiers in the early 1900s was in signal detection where crystals, vacuum tubes, or diodes served as the nonlinear element [2], [3]. An excellent discussion of the early history of microwave detectors is provided in [4]. These early microwave rectifiers were aimed at extracting information rather than extracting dc power. The first published application of microwave rectifiers for extraction of dc power was performed in the 1960s using diode-based rectifiers [5]-[8].

Renewed interest in free-space power transmission occurred in the early 1970s. An interesting microwave rectifier for production of dc power or low-frequency ac power called the cyclotron-wave rectifier was introduced in [9] and [10]. William C. Brown, Raytheon, Boston, MA, one of the original researchers in the field, continued publishing diode-based rectifier work and introduced the term "rectenna" for a receiving antenna integrated with a rectifier [11]-[13]. Around the same time, power combining for an array of microwave power rectifiers was discussed in [14], in which the authors inadvertently graze the topic of harmonically terminated rectifiers, of which they seem to hint at a class-F rectifier.

A number of diode-based rectifiers have been demonstrated, many integrated with antennas, with a good comparison presented in [15] and in earlier works focusing on low-power rectification [16], [17]. Additional applications where rectifier efficiency is important include microwave power recycling [18], and dc-dc converters with extremely high-frequency switching [19], [20]. In many of the reported microwave rectifiers, filtering of the harmonics at both the input and output has been investigated, e.g., [21] and [22], mainly to reduce re-radiated harmonic power. To date, very few transistor rectifier circuits have been demonstrated, most at frequencies at least three times lower than in this study. A UHF synchronous transistor class-E rectifier at $700 \mathrm{MHz}$ is shown to achieve $85 \%$ efficiency with $58 \mathrm{~mW}$ of output power in [20], and the same authors discuss a class- $E^{2} 10-\mathrm{W}$ dc-dc converter with a synchrounous transistor rectifier at $780 \mathrm{MHz}$ with $72 \%$ efficiency [23]. This design is scaled from $0.5-\mathrm{MHz}$ synchronous rectifier designs demonstrated in [24] and requires an additional synchronized gate RF input signal.

Harmonic terminations are commonly applied to increase efficiency in power amplifiers (PAs). The transistor nonlinearities generate harmonic content at the output, and in a number of high-efficiency amplifier classes, specific harmonic terminations are used to shape the current and voltage waveforms. In reduced conduction angle PAs (classes $\mathrm{A}, \mathrm{AB}, \mathrm{B}$, and $\mathrm{C}$ ), all harmonics are shorted at the virtual drain reference plane in the transistor. Other PA modes of operation specify open or short 


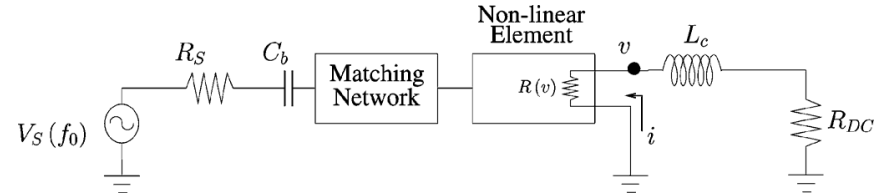

Fig. 1. Microwave rectifier circuit diagram. An ideal blocking capacitor $C_{b}$ provides dc isolation between the microwave source and rectifying element. An ideal choke inductor $L_{c}$ isolates the dc load $R_{\mathrm{DC}}$ from RF power.

harmonic terminations for various harmonics [25]-[27]. A general analysis for arbitrary complex terminations of harmonics have recently been derived in [28], including a sensitivity analysis to harmonic termination impedances. In a rectifier, the nonlinear rectifying element also generates currents and voltages at the harmonics of the input frequency, and although in this case the output is at dc, the efficiency of the rectifier can be modified by terminating the harmonics. In [29], the harmonic termination concept for improving rectifier efficiency is applied to a class-C diode rectifier integrated with a dual-polarized patch antenna for a wireless powering application.

In this paper, we identify the similarity between power rectifiers and PAs, showing that many of the efficiency improvement techniques developed for PAs may be practically directly applied to power rectifiers. Particularly, the impact that harmonic terminations have on the rectification efficiency is addressed. A general rectifier analysis approach is presented in Section II, and several classes of microwave power rectifiers are introduced, focusing on class- $\mathrm{C}$ and $\mathrm{F}^{-1}$ modes, which are experimentally validated in Sections III and IV.

\section{HaRmonically TeRminated POWER-RECTIFIER ANALYSIS}

Consider the microwave rectifier shown in Fig. 1. A sinusoidal microwave power source with voltage magnitude $V_{s}$ and impedance $R_{s}$ drives the rectifying element having a resistance $R(v)$ defined as

$$
R(v)= \begin{cases}\infty, & v>0 \\ 0, & v \leq 0\end{cases}
$$

where $v$ and $i$ are the instantaneous voltage across and current through the rectifying element, respectively. The rectifying element depicted by $R(v)$ in Fig. 1 can, in general, be any nonlinear device that acts as a switch, such as a diode or a transistor. When a nonzero on-resistance $R_{\text {on }}$ and nonzero threshold voltage $V_{\text {tr }}$ are taken into account, the resistance of the rectifying element is given by

$$
R(v)=\left\{\begin{array}{lc}
\infty, & v>-V_{\mathrm{tr}} \\
R_{\mathrm{on}}, & v \leq-V_{\mathrm{tr}}
\end{array}\right.
$$

The analysis of different classes of power rectifiers will be analyzed next based on the harmonic terminations presented to the rectifying element, and independent of the physical nonlinear device, which performs the rectification.

\section{A. Class-C Rectifier Analysis}

In Fig. 1, a sinusoidal microwave power source with voltage magnitude $V_{s}$ and impedance $R_{s}$ drives the rectifying element of resistance $R(v)$ above. The dc load seen by the rectifying element is $R_{\mathrm{DC}}$, while the load at the fundamental frequency $f_{0}$ and successive harmonics is set by the matching network. Assume the matching circuit presents $R_{s}\left(f_{0}\right)$ to the rectifying element with all subsequent harmonics terminated in short circuits. This is equivalent to the harmonic terminations for a canonical reduced conduction angle PA. This class is useful for Schottky diode rectifiers because these diodes have nonlinear junction capacitance. Short circuiting the harmonics fixes the harmonic terminations at the intrinsic diode by shorting this junction capacitance.

When the incident RF voltage at the ideal rectifier swings negative, it is clipped at zero given (1). The enforced harmonic terminations force the voltage waveform to contain only a dc and fundamental frequency component. Therefore, a dc component must be produced by the rectifying element such that the voltage waveform maintains its sinusoidal nature. The voltage across the rectifying element can now be expressed as

$$
v(\theta)=V_{\mathrm{DC}}+V\left(f_{0}\right) \sin (\theta)
$$

where $V\left(f_{0}\right)$ is the fundamental frequency component of the voltage across the rectifying element, $V_{\mathrm{DC}}$ is the dc component, $V_{\mathrm{DC}}=V\left(f_{0}\right)$, and $\theta=2 \pi f_{0} t$. The current waveform contains infinite frequency components, and can be written as

$$
i(\theta)=2 \pi I_{\mathrm{DC}} \delta\left(\theta-\frac{3 \pi}{2}-2 n \pi\right), \quad n=0,1, \ldots, \infty
$$

where $I_{\mathrm{DC}}$ is the dc current and $\delta(\theta)$ is the Dirac delta function. When all available input power $P_{\text {in }}$ is delivered to the rectifier, the fundamental frequency component of the current through the rectifying element $I\left(f_{0}\right)$ is

$$
I\left(f_{0}\right)=\frac{2 P_{\text {in }}}{V\left(f_{0}\right)}
$$

and, since there is no mechanism by which the rectifier itself can dissipate power ( $R_{\mathrm{on}}=0$ at this point), all of the available input power must be dissipated in the dc load and the conversion efficiency is $100 \%$. Therefore,

$$
P_{\mathrm{in}}=V_{\mathrm{DC}} I_{\mathrm{DC}} .
$$

Substituting in (5) and rearranging gives the expression of the current at the fundamental input frequency and the dc rectified current, which is $I\left(f_{0}\right)=2 I_{\mathrm{DC}}$. When all available input power is delivered to the rectifier, the RF-dc conversion efficiency is $100 \%$ because the rectifying element is ideal and cannot dissipate power itself. In order for all available input power to be delivered to the rectifier, it is straightforward to show that the dc load must be set relative to the fundamental frequency load as

$$
R_{\mathrm{DC}}=2 R_{s}\left(f_{0}\right) .
$$

A harmonic-balance simulation of an approximately ideal rectifier with short-circuited harmonic terminations was performed in Microwave Office using the SPICE diode model with no parasitics (PNIV) as the rectifying element. The device temperature was set to $1 \mathrm{~K}$ to approximate an ideal switch. The 


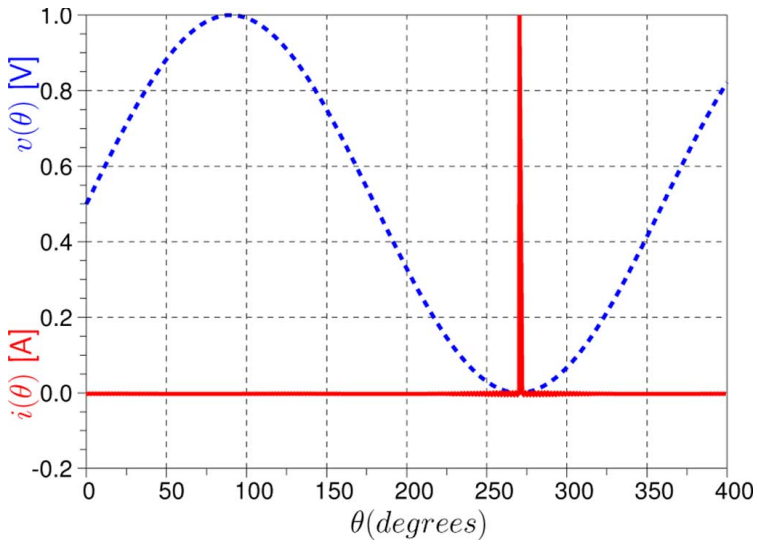

Fig. 2. Ideal normalized voltage (dashed) and current (solid) waveforms for reduced conduction angle half-wave rectifier. The waveforms have been normalized to their peak values.

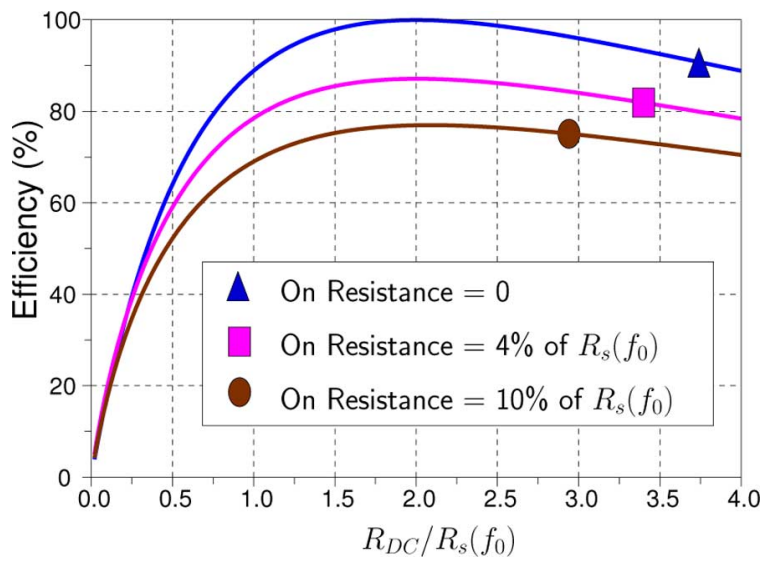

Fig. 3. Simulated efficiency of reduced conduction angle half-wave rectifier versus $R_{\mathrm{DC}} / R_{s}\left(f_{0}\right)$ for varying rectifier on-resistance.

fundamental frequency excitation was set to $1 \mathrm{~W}$ at $1 \mathrm{GHz}$ with the first 200 harmonics terminated in short circuits. The diode was presented with $50 \Omega$ at the fundamental frequency and the dc load was swept from 5 to $200 \Omega$. The simulated data is then normalized to generalize the simulation results. The ideal time-domain current and voltage waveforms across the diode are shown in Fig. 2 with the RF-dc conversion efficiency as a function of $R_{\mathrm{DC}} / R_{s}\left(f_{0}\right)$ for varying rectifier on-resistance shown in Fig. 3. It is clear that the mechanism of operation in the ideal case agrees with the theory presented above. The reduction in RF-dc conversion efficiency when the dc load is not set according to (7) is due to impedance mismatch, and is given by

$$
\eta=1-\left(\frac{R_{\mathrm{DC}}-2 R_{s}\left(f_{0}\right)}{R_{\mathrm{DC}}+2 R_{s}\left(f_{0}\right)}\right)^{2}
$$

\section{B. Class- $F^{-1}$ Rectifier Analysis}

Consider again the rectifier circuit shown in Fig. 1 and assume that all even harmonics are terminated in open circuits, while all odd harmonics are terminated in short circuits. This set of harmonic terminations is the same as for a class- $\mathrm{F}^{-1}$ amplifier, therefore this rectifier will be referred to as a class- $\mathrm{F}^{-1}$ rectifier. The fundamental frequency component of the voltage across the rectifying device is given by

$$
V\left(f_{0}\right)=V_{s}\left(f_{0}\right) .
$$

During the second half of the RF cycle, it is evident from (1) that the voltage across the rectifying element must be zero. This condition must be met through the addition of dc and only even harmonic voltage components, and therefore the voltage waveform is expressed as

$$
v(\theta)=\left\{\begin{array}{l}
2 V\left(f_{0}\right) \sin \theta, \quad 0 \leq \theta<\pi \\
0, \quad \pi \leq \theta<2 \pi .
\end{array}\right.
$$

A Fourier expansion of (10) expresses the dc component of the voltage waveform as

$$
V_{\mathrm{DC}}=\frac{2 V\left(f_{0}\right)}{\pi} .
$$

In the first half of the RF cycle, the current through the rectifying element is zero, given (1). This condition is met through the addition of a de current and odd harmonic current components. With the current direction as in Fig. 1, the dc component of the current must be positive. Therefore, in the first half of the RF cycle, the remaining harmonics must sum to a constant value equivalent to the negative of the dc component. Since the function, which is the sum of the remaining harmonics is odd, the second half of the RF cycle must sum to the dc component, and the current is given by

$$
i(\theta)=\left\{\begin{array}{lr}
0, & 0 \leq \theta<\pi \\
2 I_{\mathrm{DC}}, & \pi \leq \theta<2 \pi .
\end{array}\right.
$$

The dc component of the current waveform Fourier expansion is found to be

$$
I_{\mathrm{DC}}=\frac{\pi}{4} I\left(f_{0}\right) .
$$

The dc load consistent with (10) and (12) is given by

$$
R_{\mathrm{DC}}=\frac{8 V\left(f_{0}\right)}{\pi^{2} I\left(f_{0}\right)}=\frac{8}{\pi^{2}} R\left(f_{0}\right) .
$$

The conversion efficiency, defined as the ratio of the dc power dissipated in the load resistor to the available fundamental frequency RF power, is evaluated as

$$
\eta=\frac{P_{\mathrm{DC}}}{P\left(f_{0}\right)}=\frac{2 V_{\mathrm{DC}} I_{\mathrm{DC}}}{V\left(f_{0}\right) I\left(f_{0}\right)}=\frac{2 \frac{2}{\pi} V\left(f_{0}\right) \frac{\pi}{4} I\left(f_{0}\right)}{V\left(f_{0}\right) I\left(f_{0}\right)}=1 .
$$

Therefore, the ideal half-wave rectifier converts all available $\mathrm{RF}$ power to dc power if the dc loading resistance is set to the value given in (14). The RF-dc conversion efficiency as a function of $R_{\mathrm{DC}} / R_{s}\left(f_{0}\right)$ was simulated in Microwave Office for varying rectifier on-resistance and is shown in Fig. 4. The harmonic-balance settings were identical to those used for the class-C rectifier above. The peak efficiency as a function of on-resistance is higher than for the class-C rectifier, although the efficiency degrades more quickly when the nonideal dc load is applied. 


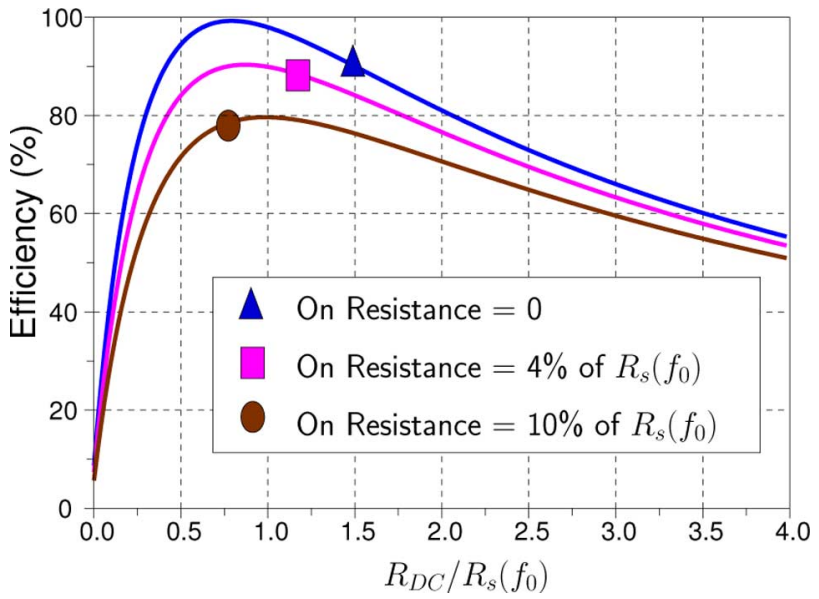

Fig. 4. Simulated efficiency of class- $\mathrm{F}^{-1}$ rectifier versus $R_{\mathrm{DC}} / R_{s}\left(f_{0}\right)$ for varying rectifier on-resistance.

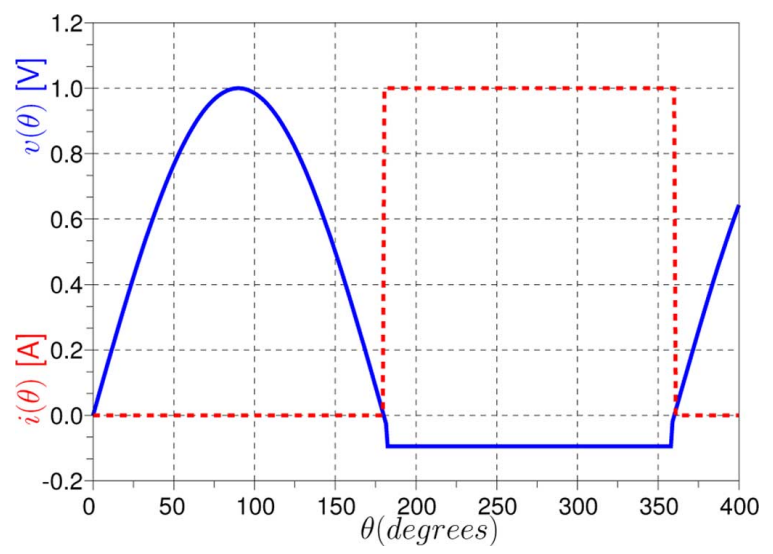

Fig. 5. Nonideal class- $\mathrm{F}^{-1}$ voltage (solid) and current (dashed) waveforms, normalized to their peak respective values.

The waveforms including parasitic on-resistance and threshold voltage are next investigated assuming the rectifier impedance from (2). The time-domain voltage and current waveforms are approximated as

$$
\begin{aligned}
& v(\theta)=\left\{\begin{array}{l}
V_{\max } \sin \theta, \quad v(\theta)>-V_{\mathrm{tr}} \\
-V_{\mathrm{tr}}-I_{\max } R_{\mathrm{on}}, \quad v(\theta) \leq-V_{\mathrm{tr}}
\end{array}\right. \\
& i(\theta)= \begin{cases}0, & V(\theta)>-V_{\mathrm{tr}} \\
I_{\max }, \quad v(\theta) \leq-V_{\mathrm{tr} .}\end{cases}
\end{aligned}
$$

As an example, Fig. 5 shows the current and voltage waveforms for a specific set of nonideal parameters $\left(V_{\mathrm{tr}}=0.7 \mathrm{~V}\right.$, $V_{\max }=20 \mathrm{~V}, I_{\max }=200 \mathrm{~mA}$, and $R_{\mathrm{on}}=5 \Omega$ ). When the device is conducting current, it creates a voltage drop across the on-resistance, which is constant due to the constant current. If the on-resistance were zero, the only difference between the waveform in (16) and the ideal voltage waveform would be the minimum value, which would be $-V_{\mathrm{tr}}$ rather than zero.

The values of $\theta$ at which the transition between the conducting and nonconducting regions occurs are found to be

$$
\begin{aligned}
& \theta_{t 1}=2 \pi-\arcsin \left(\frac{V_{\mathrm{tr}}}{V_{\text {max }}}\right) \\
& \theta_{t 2}=\pi+\arcsin \left(\frac{V_{\mathrm{tr}}}{V_{\max }}\right) .
\end{aligned}
$$

The dc and fundamental frequency values of the voltage and current waveforms can be found through a Fourier analysis using the transition points in (18). The first Fourier coefficient of $v(t)$ gives the dc component of the voltage, which can be derived as

$$
\begin{aligned}
V_{\mathrm{DC}}=\frac{1}{2 \pi}\left(2 V_{\max } \sqrt{1-\left(\frac{V_{\mathrm{tr}}}{V_{\max }}\right)^{2}}\right. \\
\left.\quad-\left(V_{\mathrm{tr}}+I_{\max } R_{\mathrm{on}}\right)\left[\pi-2 \arcsin \left(\frac{V_{\mathrm{tr}}}{V_{\max }}\right)\right]\right) .
\end{aligned}
$$

The fundamental frequency voltage is found from $V\left(f_{0}\right)=$ $a_{v}+j b_{v}$, where

$$
a_{v}=\frac{1}{\pi} \int_{0}^{2 \pi} v(\theta) \cos \theta d \theta=0
$$

and $b_{v}$ can be reduced to

$$
\begin{aligned}
b_{v}=\frac{1}{\pi}\left(V_{\max }\right. & \arcsin \left(\frac{V_{\mathrm{tr}}}{V_{\max }}\right)+\frac{\pi V_{\max }}{2} \\
& \left.+\left(V_{\mathrm{tr}}+2 I_{\max } R_{\mathrm{on}}\right) \sqrt{1-\left(\frac{V_{\mathrm{tr}}}{V_{\mathrm{max}}}\right)^{2}}\right) .
\end{aligned}
$$

Similarly, the dc component of the current waveform is found to be

$$
I_{\mathrm{DC}}=\frac{I_{\max }}{2 \pi}\left(\pi-2 \arcsin \left(\frac{V_{\mathrm{tr}}}{V_{\max }}\right)\right) .
$$

The fundamental frequency current $i(t)=a_{i}+j b_{i}$ has $a_{i}=0$ and the coefficient $b_{i}$ can be shown to be equal to

$$
b_{i}=-\frac{2 I_{\mathrm{max}}}{\pi} \sqrt{1-\left(\frac{V_{\mathrm{tr}}}{V_{\max }}\right)^{2}} .
$$

The input power at the fundamental frequency is found from

$$
P_{\mathrm{in}}=\Re\left\{\frac{V\left(f_{0}\right) I^{*}\left(f_{0}\right)}{2}\right\} .
$$

Substituting (21) and (23) into the above results in

$$
\begin{aligned}
& P_{\mathrm{in}}=\frac{k I_{\mathrm{max}}}{\pi^{2}}\left(V_{\max } \arcsin (\right.\left.\frac{V_{\mathrm{tr}}}{V_{\mathrm{max}}}\right)+\frac{\pi V_{\max }}{2} \\
&\left.+\left(V_{\mathrm{tr}}+2 I_{\max } R_{\mathrm{on}}\right) k\right)
\end{aligned}
$$

where $k$ is defined as

$$
k=\sqrt{1-\left(\frac{V_{\mathrm{tr}}}{V_{\max }}\right)^{2}} .
$$

Solving for $I_{\max }$ as a function of $P_{\text {in }}$ when $R_{\text {on }}$ is nonzero after some arithmetic results in two solutions, one of which is negative. The positive solution for the maximal current is

$$
I_{\max }=\frac{\sqrt{\alpha^{2}+8 \pi^{2} P_{\mathrm{in}} R_{\mathrm{on}}}-\alpha}{4 R_{\mathrm{on}} k}
$$

with $\alpha=V_{\max }\left(\arcsin \left(V_{\mathrm{tr}} / V_{\max }\right)+\pi / 2+k V_{\mathrm{tr}} / V_{\max }\right)$. 
In the case where $R_{\text {on }}$ is zero, (27) simplifies to

$$
I_{\max }=P_{\mathrm{in}} \frac{\pi^{2}}{k V_{\max }\left(\arcsin \left(\frac{V_{\mathrm{tr}}}{V_{\max }}\right)+\frac{\pi}{2}+k \frac{V_{\mathrm{tr}}}{V_{\max }}\right)} .
$$

Note that in the case of an ideal rectifying element, $k=1$ and $V_{\mathrm{tr}}=0$, therefore,

$$
I_{\text {max }, \text { ideal }}=\frac{2 \pi P_{\text {in }}}{V_{\max }} .
$$

Now that $I_{\max }$ is fully expressed given known rectifier parameters, $V_{\mathrm{DC}}$ and $I_{\mathrm{DC}}, V\left(f_{0}\right)$ and $I\left(f_{0}\right)$ may be calculated, and from this, the dc load and the load at fundamental frequency determined from the following expressions:

$$
\begin{aligned}
R_{\mathrm{DC}} & =\frac{V_{\mathrm{DC}}}{I_{\mathrm{DC}}} \\
R\left(f_{0}\right) & =-\frac{V\left(f_{0}\right)}{I\left(f_{0}\right)}=-\frac{b_{v}}{b_{i}} .
\end{aligned}
$$

The negative impedance in (31) indicates that power is delivered to the rectifying element and gives the impedance of the source delivering power to the rectifying element. The rectifier efficiency is given by

$$
\eta=\frac{P_{\mathrm{DC}}}{P_{\mathrm{in}}}=\frac{V_{\mathrm{DC}} I_{\mathrm{DC}}}{P_{\text {in }}} .
$$

\section{Design Example Based on Class $-F^{-1}$ Theory}

To understand the usefulness of the presented theory, assume the rectifying element has the following parameters: $V_{\max }=$ $10 \mathrm{~V}, R_{\text {on }}=5 \Omega, V_{\mathrm{tr}}=0.7 \mathrm{~V}$, and $P\left(f_{0}\right)=1 \mathrm{~W}$. First, (27) is used to calculate $I_{\max }=456.7 \mathrm{~mA}$. Next, the dc voltage and current are evaluated using (19) and (22), respectively, to give $V_{\mathrm{DC}}=1.75 \mathrm{~V}$ and $I_{\mathrm{DC}}=218.2 \mathrm{~mA}$. The fundamental frequency voltage and current Fourier coefficients are then calculated to be $V\left(f_{0}\right)=6.896 \mathrm{~V}$ and $I\left(f_{0}\right)=-290 \mathrm{~mA}$, respectively. The dc and fundamental frequency resistances are then calculated using (30) and (31) to be $R_{\mathrm{DC}}=8.02 \Omega$ and $R\left(f_{0}\right)=23.77 \Omega$, respectively. The efficiency is then calculated using (32) to be $\eta=38.18 \%$. If the input power is selected as $0.1 \mathrm{~W}$ rather than $1 \mathrm{~W}$, the resulting efficiency is $72.43 \%$. A specific rectification device will always have an approximate input drive level at which it can be most efficient, just as with power transistors in PAs. To maximize efficiency, the goal is always to minimize the amount of power dissipation in the on-resistance of the rectifying element and maximize the power dissipated in the dc load resistor.

\section{SCHOTTKY-DiOde CLASS-C RECTIFIER}

The Skyworks SMS7630 Schottky diode in the SC-79 package was selected for the half-wave rectifier. Source-pull was performed at $2.45 \mathrm{GHz}$ with $0-10 \mathrm{dBm}$ available input power for various de loads in order to identify the combination of input power, fundamental load, and dc load resulting in highest efficiency. The best case occurred at 6-dBm input

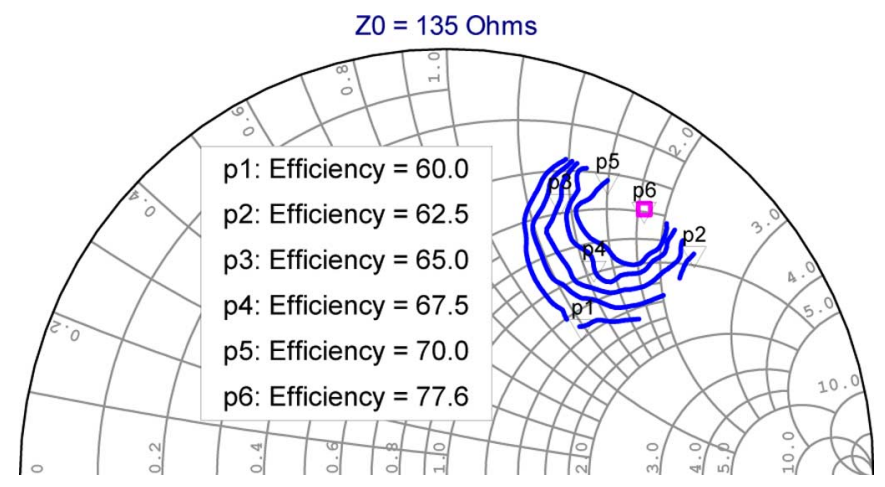

Fig. 6. Source-pull contours with available input power to the diode set to $6 \mathrm{dBm}$. The impedance is referenced to the junction capacitance of the diode, therefore the lead inductance of the package has been compensated for. Setting $R_{\text {DC }}$ to $1080 \Omega$ was found to result in the optimal efficiency for this input power. The highest efficiency of $77.6 \%$ is obtained at $Z_{p 6}=(68+j 245) \Omega$ with $V_{\mathrm{DC}}=1.82 \mathrm{~V}$.

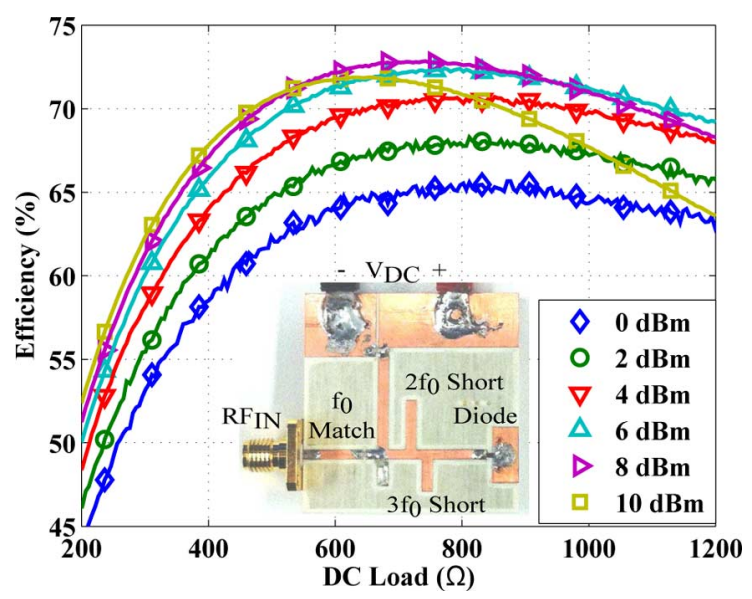

Fig. 7. RF-dc conversion efficiency versus de load for fixed available input powers with $0.6-\mathrm{dB}$ matching network loss de-embedded. The maximum efficiency of $72.8 \%$ occurred at $8 \mathrm{dBm}$ with $R_{\mathrm{DC}}=742 \Omega$ and $V_{\mathrm{DC}}=1.91 \mathrm{~V}$, which is lower than the $1080 \Omega$ found during source-pull. However, the efficiency at $1080 \Omega$ is $69.9 \%$ which is very close to the peak value.

power with the source-pull contours shown in Fig. 6. The on-resistance of the SMS7630 is $20 \Omega$ with the optimal dc load of $1080 \Omega$. Therefore, $R_{\text {on }}$ is approximately $2 \%$ of $R_{\mathrm{DC}}$, which, in theory, is $4 \%$ of $R_{s}\left(f_{0}\right)$. From Fig. 3, a peak efficiency of $87 \%$ occurs with infinite harmonic terminations, therefore the achieved $77.6 \%$ is very reasonable considering only the second and third harmonics were explicitly terminated.

Measurements of a rectifier designed using the source-pull data show a maximum RF-dc conversion efficiency of $72.8 \%$ when matched to $50 \Omega$, obtained after the 0.6 -dB matching network loss is de-embedded. The fabricated rectifier and dc load sweep measurements are shown in Fig. 7. Open-circuit shunt stubs are used to present short-circuit terminations at the second and third harmonics. A shunt capacitor is used for presenting the fundamental frequency impedance to reduce size and allow tunability. The reduction in efficiency relative to the source-pull measurements is due to the matching circuit not presenting the ideal impedance found during source-pull. 


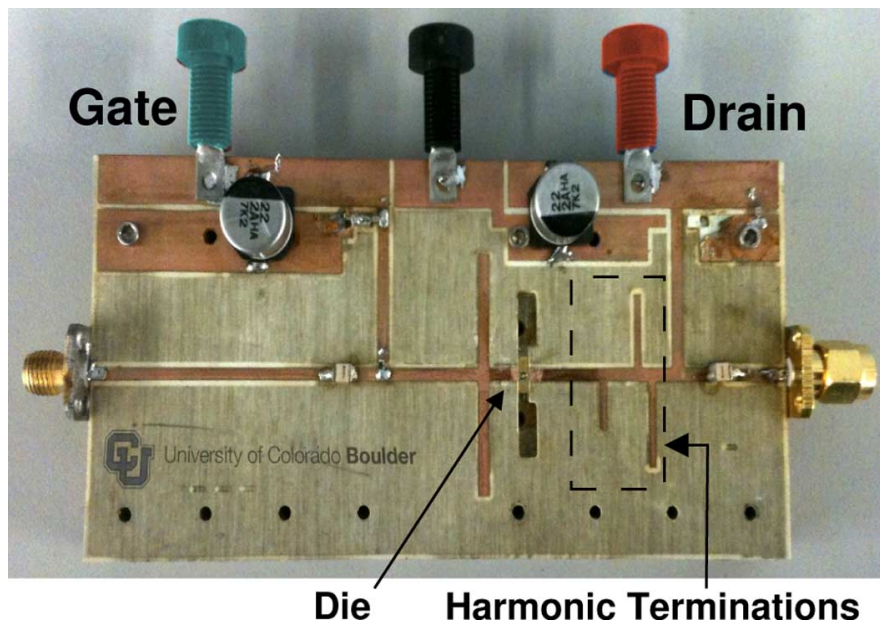

Fig. 8. Photograph of the class- $\mathrm{F}^{-1} \mathrm{PA}$, working at $2.14 \mathrm{GHz}$ and presented in [30].

The class-C rectifier can be applied to improving the efficiency of a wireless powering reception device, as demonstrated in [29], with a dual-linearly polarized patch rectenna, with a rectifier circuit for each polarization. In this circuit, the first five harmonics are shorted and the impedances are validated by calibrated measurements and are presented in [29].

\section{TRANSISTOR Class-F ${ }^{-1}$ RECTIFIER}

To prove experimentally the duality between harmonically terminated PAs and rectifiers, a high-efficiency class- $\mathrm{F}^{-1}$ PA was designed, measured first as an amplifier, and then as a rectifier. In the rectifier measurements, RF power is input into the drain, which is unbiased. The gate is terminated in a variable impedance and biased close to pinch-off. Measurements of efficiency and dc voltage are performed in the time domain as a function of input RF power, gate RF load, gate bias, and drain dc load.

\section{A. Circuit Design}

A 2.14-GHz PA, shown in Fig. 8, is designed using the Triquint TGF2023-02 GaN HEMT [30]. Class $\mathrm{F}^{-1}$ harmonic terminations are implemented at the second and third harmonics. The performance of the PA, illustrated in Fig. 9, was characterized at $2.14 \mathrm{GHz}$ with a drain voltage bias of $28 \mathrm{~V}$ and a bias current of $160 \mathrm{~mA}$. The PA exhibits a power-added efficiency (PAE) of $84 \%$ with an output power of $37.6 \mathrm{dBm}$ and a gain of $15.7 \mathrm{~dB}$ under $3-\mathrm{dB}$ compression. The same PA design was used for rectifier measurements, as shown in Fig. 10. The PA is connected to an input RF source at the drain with the drain supply disconnected. The gate terminal is biased, and connected to an impedance tuner, converting the two-port transistor PA to a one-port rectifier, corresponding to the generalized schematic of Fig. 1.

\section{B. Measurement Setup}

The class- $\mathrm{F}^{-1} \mathrm{PA}$ described above is fully characterized in large signal in a rectifier configuration with the setup shown

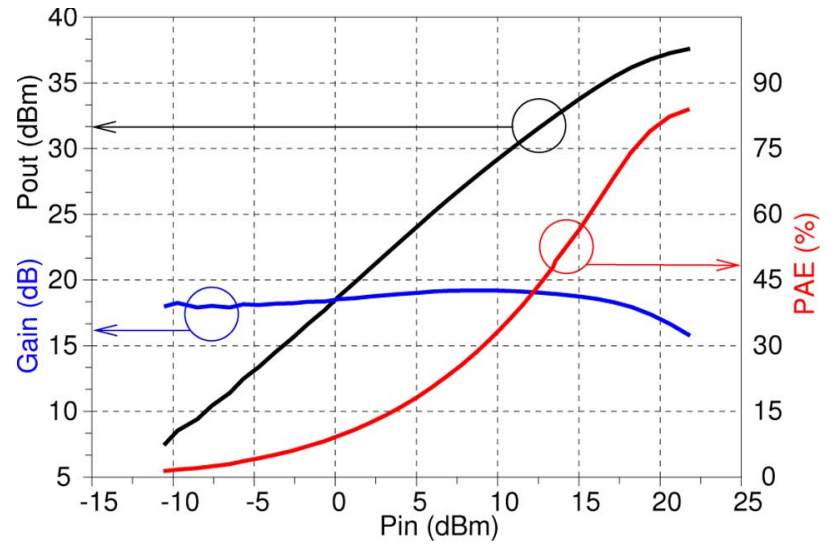

Fig. 9. Large-signal measurements performed on the class- $\mathrm{F}^{-1} \mathrm{PA}$ at $f_{0}=$ $2.14 \mathrm{GHz}, V_{\mathrm{GS}}=-3.8 \mathrm{~V}$, and $V_{\mathrm{DS}}=28 \mathrm{~V}$.

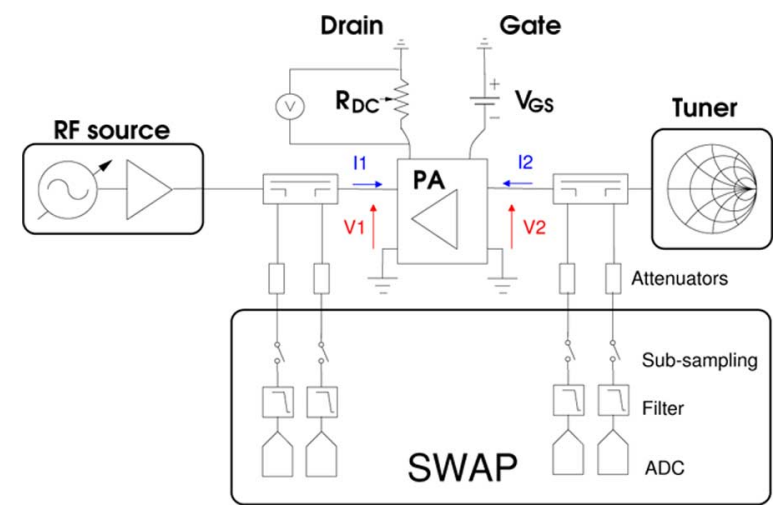

Fig. 10. Time-domain nonlinear rectifier measurement block diagram. The SWAP [31] performs sampling of current and voltage and the calibration refers the sampled quantities to the reference planes at the DUT. The drain output dc resistance $R_{\mathrm{DC}}$, the gate bias $V_{\mathrm{GS}}$, and the gate RF impedance $Z_{g}$ are varied as the input power at the drain is swept from 10 to $42 \mathrm{dBm}$.

in Fig. 10. The commercial time-domain large-signal measurement instrument is a VTD SWAP four-channel receiver [31]. In order to acquire time-domain waveforms at the reference plane, an eight-error term model calibration similar to the one performed for large-signal network analyzer (LSNA) measurements is applied. After an absolute vector network analyzer (VNA)-like calibration [32], the RF voltage and current waveforms at the input (V1 and I1) and at the output (V2 and I2) of the device-under-test (DUT) are measured at the coaxial reference plane. In this case, the RF input is the drain port of the PA, while the RF output is connected to the gate port. Thus, performing a load-pull on this device consists of varying the load at $f_{0}$ at the RF gate port of the PA with a passive tuner. This kind of measurement is similar to large-signal characterization of switch devices recently reported in [33] and [34]. The gate dc path is connected to a power supply so the gate bias can be varied. The drain dc bias is the output of the rectifier and is connected to a variable resistance $R_{\mathrm{DC}}$, and the dc voltage across it is measured with a voltmeter. The dc current is then found from the value of $R_{\mathrm{DC}}$ from (30). During the measurement, several parameters are varied systematically: the RF load impedance applied at the PA gate port $Z_{g}\left(f_{0}\right)=V_{g}\left(f_{0}\right) / I_{g}\left(f_{0}\right)$; the resistor in the dc drain output $R_{\mathrm{DC}}$; and the gate bias voltage $V_{\mathrm{GS}}$. 


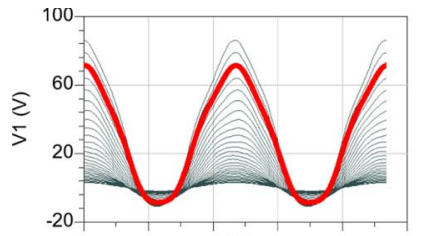

(a)
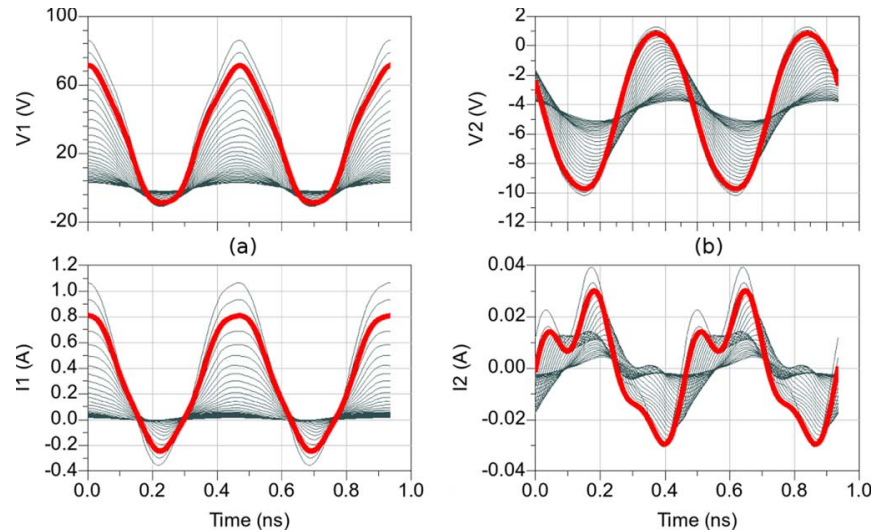

(b)

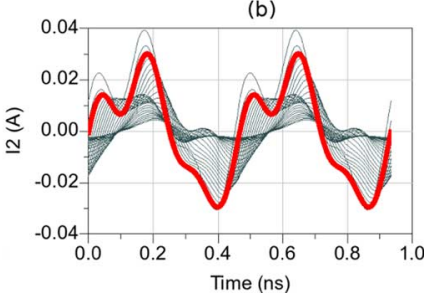

Fig. 11. Time-domain waveforms measured at: (a) drain and (b) gate of the rectifier with $V_{\mathrm{GS}}=-4.4 \mathrm{~V}, R_{\mathrm{DC}}=98.5 \Omega$, and $Z_{g}\left(f_{0}\right)=(230+j 10) \Omega$. The RF input power at the drain is swept from 10 to $42 \mathrm{dBm}$, corresponding to the range of output power of the class-F -1 PA.

The conversion efficiency of the rectifier and the dc power delivered at the drain output of the rectifier $P_{\mathrm{DC}}=V_{\mathrm{DC}} \cdot I_{\mathrm{DC}}$ are measured as these parameters are varied, and as a function of input power at the drain port $P_{\text {in }}\left(f_{0}\right)$.

\section{Self-Synchronous Transistor Rectifier Results}

The measurements of the rectifier are performed in self-synchronous mode, i.e., there is no input RF power incident externally into the gate port of the PA, unlike in previous transistor rectifier work [20], [24]. The following parameters are varied in order, while keeping the other parameters constant and sweeping the input RF power at the drain port, and the results are described in the same order:

1) RF impedance at the gate, $Z_{g}$;

2) load resistance at drain bias output, $R_{\mathrm{DC}}$;

3) gate dc bias, $V_{\mathrm{GS}}$.

The gate load-pull was performed to determine the optimum impedance for maximum efficiency with a constant resistive dc load of $98.5 \Omega$ (nominally $100 \Omega$ ) and a constant transistor gate bias in pinch-off of $-4.4 \mathrm{~V}$. The RF signal is coupled from the drain to the gate matching network through the feedback capacitance $C_{g d}$, and thus the precise impedance presented at the gate of the transistor is imperative to achieving high efficiency. Fig. 11 shows the time-domain voltage and current waveforms measured at the drain and gate RF port of the amplifier when the $\mathrm{RF}$ input power at the drain port is swept from 11 to $42 \mathrm{dBm}$. These values are chosen because the rectifier in PA operation gives up to 42-dBm output power. The feedback signal present at the gate allows for the rectifier to operate in self-synchronous mode without any additional control signal. Unlike in the synchronously driven case where an external generator is connected to the gate, here the impedance presented at the gate is always passive (inside the Smith chart), keeping the device in a safe operating mode.

Measured RF-dc conversion efficiency is shown in Fig. 12 for four different RF gate impedances. A maximal conversion efficiency of $85 \%$ is achieved with a dc output voltage of $36 \mathrm{~V}$ and an input power at the drain of $42 \mathrm{dBm}$ with $R_{\mathrm{DC}}=98.5 \Omega$. This peak efficiency is for a RF gate load of around $230 \Omega$ (green hexagon (in online version) in the Smith chart in Fig. 12), which
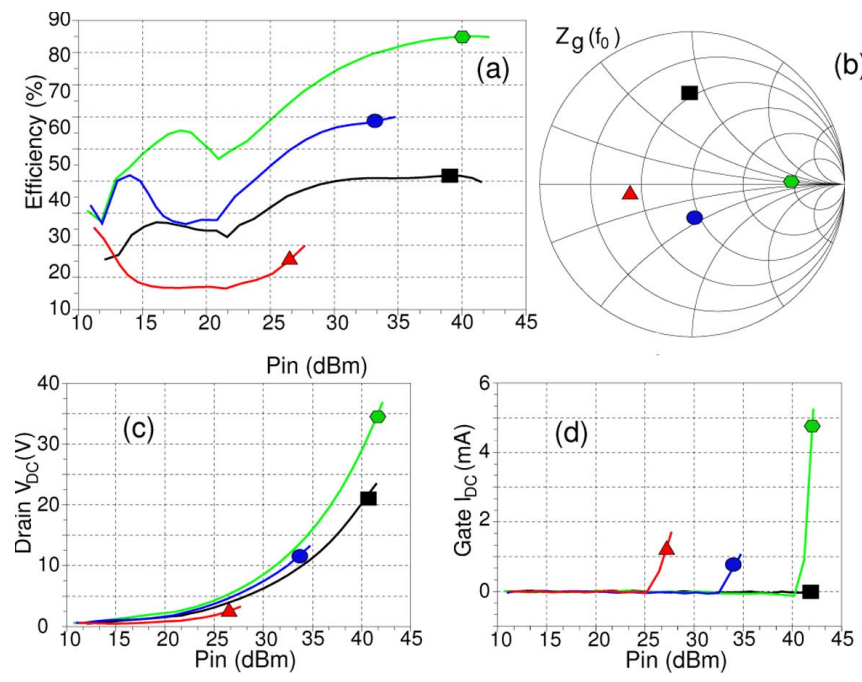

Fig. 12. Conversion efficiency, gate dc current, and drain dc voltage versus input power for several RF load impedance values presented at the gate. $V_{\mathrm{GS}}=$ $-4.4 \mathrm{~V}$ and $R_{\mathrm{DC}}=98.5 \Omega$. The green point (in online version) on the Smith chart corresponds to the highest efficiency point at $Z_{g}\left(f_{0}\right)=(230+j 10) \Omega$.

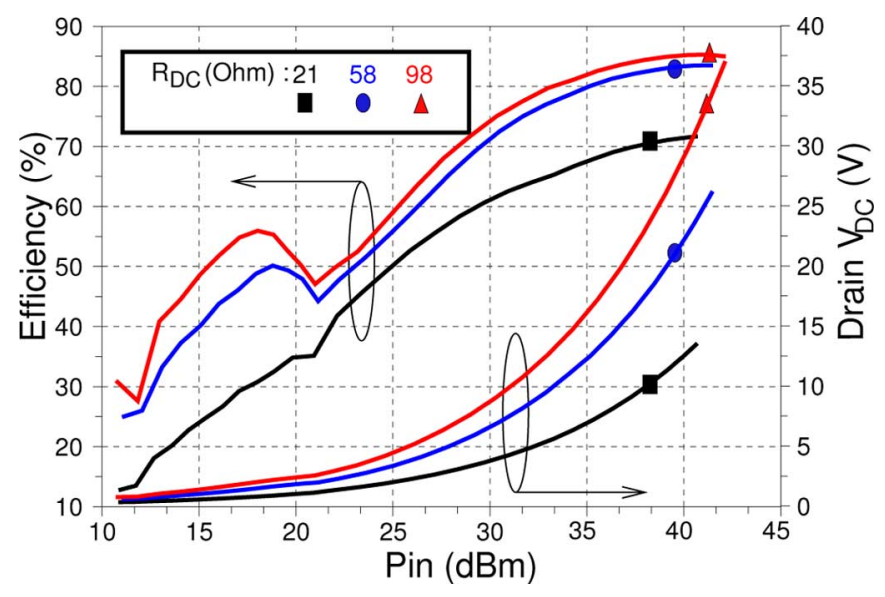

Fig. 13. Conversion efficiency and drain dc output voltage versus input power for several dc drain resistor values. $V_{\mathrm{GS}}=-4.4 \mathrm{~V}$ and $Z_{g}\left(f_{0}\right)=(230+j 10) \Omega$. The highest efficiency of $85 \%$ is obtained at $P_{\text {in }}=40 \mathrm{dBm}$ with a $V_{\mathrm{DC}}=30 \mathrm{~V}$.

is the highest impedance that was achievable with the specific tuner in the setup. For the low gate impedance (red triangle (in online version) in the Smith chart), the efficiency is significantly lower. By observing the gate current [see Fig. 12(d)], it can be seen that, for a low RF gate impedance, the gate diode turns on at around $P_{\text {in }}=25 \mathrm{dBm}$. Since the input power cannot be increased much beyond this point to avoid breakdown, this limits the dc voltage at the output to around $4 \mathrm{~V}$. For the gate impedance with highest efficiency (green line (in online version) with hexagon symbol), the gate diode is off for input drain powers below $41 \mathrm{dBm}$, allowing for high de voltage output.

After the optimal gate impedance for highest efficiency was obtained, a power sweep for three different $R_{\mathrm{DC}}$ values in the drain output was obtained. From Fig. 13, a maximal efficiency of $85 \%$ was measured for a de resistive load of $98 \Omega$, while an efficiency drop of $13 \%$ was observed for a de load of $21 \Omega$ with $40-\mathrm{dBm}$ input power. As expected, the dc output voltage decreases from a maximum $30 \mathrm{~V}$ for $R_{\mathrm{DC}}=98 \Omega$ at $40-\mathrm{dBm}$ input 


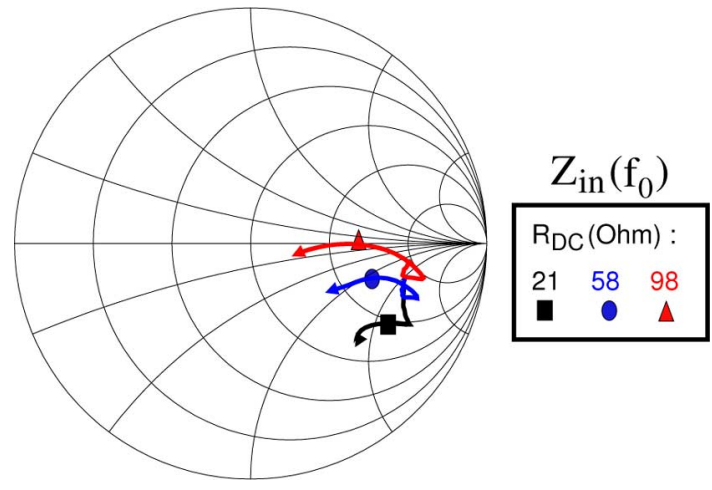

Fig. 14. RF impedance at $f_{0}$ measured at the input (drain port) versus input power for several dc drain resistor values. $V_{\mathrm{GS}}=-4.4 \mathrm{~V}$ and $Z_{g}\left(f_{0}\right)=$ $(230+j 10) \Omega$.

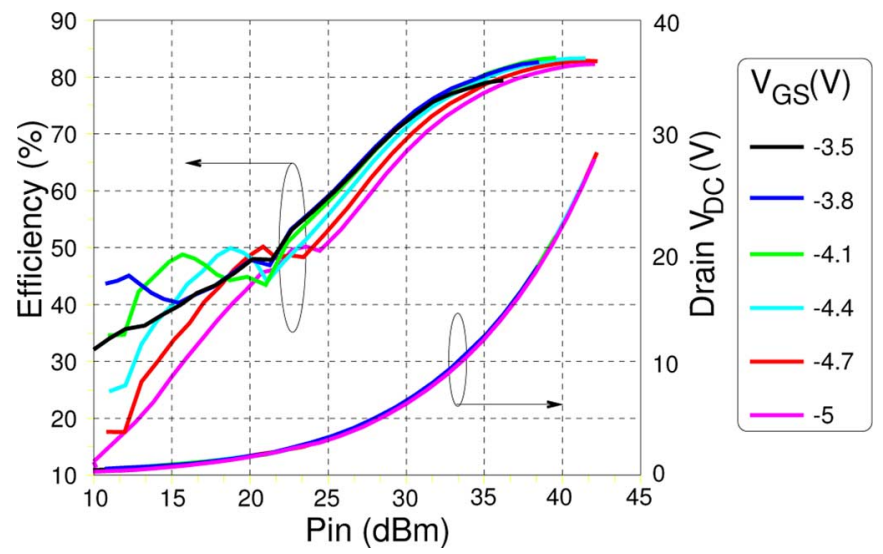

Fig. 15. Measured conversion efficiency and drain dc voltage versus input power for several dc gate voltage biases. For this data, $R_{\mathrm{DC}}=58 \Omega$ and $Z_{g}\left(f_{0}\right)=(230+j 10) \Omega$.

power, to a maximum of $13.4 \mathrm{~V}$ for $R_{\mathrm{DC}}=21 \Omega$ with the same input power. It is interesting to see how the input impedance of the rectifier at the RF drain port approaches $50 \Omega$ as the input power increases (Fig. 14). This is expected since the PA was designed for maximal saturated power delivered into a $50-\Omega$ load. This again points to the similarities between the same circuit operated as a power rectifier and a PA.

Finally, the effect of the gate bias $V_{\mathrm{GS}}$ on the rectifier efficiency, output voltage, and input impedance was investigated. The gate impedance in this case was set for highest efficiency $(230 \Omega)$, and a dc load of $58 \Omega$ was selected in order to protect the transistor from high drain voltages that occur for the $98-\Omega$ load that corresponds to the highest efficiency. The measurements were performed for six different values of gate bias $V_{\mathrm{GS}}$, as shown in Fig. 15. With $R_{\mathrm{DC}}=58 \Omega$, a maximum efficiency of $83 \%$ was obtained with the transistor biased deeply into the pinch-off region with $V_{\mathrm{GS}}=-4.4 \mathrm{~V}$, and a drop of only $3 \%$ was measured for $V_{\mathrm{GS}}=-3.5 \mathrm{~V}$. Furthermore, the gate bias has a minimal impact on the output dc voltage or on the drain impedance.

\section{CONCLUSiON}

In summary, this paper has addressed high-efficiency power rectifiers designed with harmonic terminations at the RF input in analogy to high-efficiency PA design with harmonic terminations at the output. The applications of such power rectifiers include wireless power beaming [7], recycling power in highpower circuits [18], and ultra-fast switching integrated dc-dc converters with no magnetics [19].

The theory for an ideal rectification element is based on Fourier analysis and establishes the basic design parameters such as the relationship between output dc resistance and impedance at the fundamental frequency at the rectifier input that optimizes efficiency. The analysis also predicts the time-domain waveforms at the terminals of the rectification element and the efficiency as a function of on-resistance and dc output resistance. Specific results are derived for class-C and class- $\mathrm{F}^{-1}$ classes of operation, as they are defined for PAs. These two cases are chosen for experimental validation with a 2.45-GHz diode and 2.14-GHz transistor rectifier, respectively. It is straightforward to repeat the derivation for other classes of operation, such as class-F, as shown in detail in [35].

The experimental results show that good agreement can be reached between theory and experiment with a Schottky-diode single-ended rectifier with finite class-C harmonic terminations, resulting in $72.8 \%$ efficiency for input power levels in the milliwatt range, intended for wireless power harvesting detailed in [15] and [29]. A GaN HEMT class- $\mathrm{F}^{-1}$ power rectifier achieved $85 \%$ efficiency with $40-\mathrm{dBm}$ input power across $98-\Omega$ dc load with a dc output voltage $V_{\mathrm{DC}}=30 \mathrm{~V}$. The efficiency and output voltage of the self-synchronous rectifier are shown to depend on the input power at the drain, the impedance at the gate port and the dc load at the output drain bias line, but not on the gate bias.

Time-domain large-signal measurements of a class- $\mathrm{F}^{-1} \mathrm{PA}$ configured as a rectifier show that one can accomplish the same rectifier efficiency as the amplifier drain efficiency in self-synchronous mode without external gate RF drive. This is somewhat surprising, and to the best of our knowledge, the first time this type of high-efficiency rectifier has been demonstrated.

\section{ACKNOWLEDGMENT}

The authors would like to thank Dr. D. Root and Dr. J.-P. Teyssier, both with Agilent Technologies, Santa Clara, CA, for the loan of the time-domain nonlinear measurement equipment and TriQuint Semiconductor, Richardson, TX, for the donation of the transistors.

\section{REFERENCES}

[1] T. C. Martin and N. Tesla, The Inventions, Researches and Writing of Nikola Tesla: With Special Reference to His Work in Polyphase Currents and High Potential Lighting. Milwaukee, WI: Lee Eng. Company, 1952.

[2] E. Peterson and F. Llewellyn, "The operation of modulators from a physical viewpoint," Proc. IRE, vol. 18, no. 1, pp. 38-48, Jan. 1930.

[3] J. Nelson, "Some notes on grid circuit and diode rectification," Proc. IRE, vol. 20, no. 6, pp. 989-1003, Jun. 1932.

[4] C. Edwards, "Microwave converters," Proc. IRE, vol. 35, no. 11, pp. 1181-1191, Nov. 1947.

[5] R. George and E. Sabbagh, "An efficient means of converting microwave energy to dc using semiconductor diodes," Proc. IEEE, vol. 51, no. 3, pp. 530-530, Mar. 1963.

[6] W. Brown, "Experiments in the transportation of energy by microwave beam," in IRE Int. Convention Rec., Mar. 1964, vol. 12, pp. 8-17. 
[7] W. Brown, J. Mims, and N. Heenan, "An experimental microwavepowered helicopter," in IRE Int. Convention Rec., Mar. 1965, vol. 13, pp. 225-235.

[8] O. E. Maynard, W. C. Brown, A. Edwards, J. T. Haley, G. Meltz, J. M. Howell, and A. Nathan, "Microwave power transmission studies. volume 1: Executive summary," NASA, Tech. Rep. 19760008506, 1975.

[9] D. Watson, K. Talbot, and C. Johnson, "A cyclotron-wave microwave power converter," Proc. IEEE, vol. 53, no. 11, pp. 1797-1798, Nov. 1965.

[10] D. Watson, R. Grow, and C. Johnson, "A cyclotron-wave rectifier for s-band and x-band," IEEE Trans. Electron Devices, vol. 18, no. 1, pp. 3-11, Jan. 1971 .

[11] W. C. Brown, "High power, efficient, free-space microwave power transmission systems," in 3rd Eur. Microw. Conf., Sep. 1973, vol. 2, pp. 1-4.

[12] W. Brown, "The design of large scale terres trial rectennas for low-cost production and erection," in IEEE-MTT-S Int. Microw. Symp. Dig., Jun. 1978, pp. 349-351.

[13] W. Brown, "The history of power transmission by radio waves," IEEE Trans. Microw. Theory Techn., vol. MTT-32, no. 9, pp. 1230-1242, Sep. 1984.

[14] R. Gutmann and J. Borrego, "Power combining in an array of microwave power rectifiers," IEEE Trans. Microw. Theory Techn., vol. MTT-27, no. 12, pp. 958-968, Dec. 1979.

[15] E. Falkenstein, M. Roberg, and Z. Popovic, "Low-power wireless power delivery," IEEE Trans. Microw. Theory Techn., vol. 60, no. 7, pp. 2277-2286, Jul. 2012.

[16] J. Hagerty, F. Helmbrecht, W. McCalpin, R. Zane, and Z. Popovic, "Recycling ambient microwave energy with broadband rectenna arrays," IEEE Trans. Microw. Theory Techn., vol. 52, no. 3, pp. 1014-1024, Mar. 2004.

[17] E. Falkenstein, "Characterization and design of a low-power wireless power delivery system," Ph.D. dissertation, Dept. Elect. Comput. Eng., Univ. Colorado at Boulder, Boulder, CO, 2011.

[18] X. Zhang, L. Larson, P. Asbeck, and R. Langridge, "Analysis of power recycling techniques for $\mathrm{rf}$ and microwave outphasing power amplifiers," IEEE Trans. Circuits Syst. II, Analog Digit. Signal Process., vol. 49, no. 5, pp. 312-320, May 2002.

[19] S. Djukic, D. Maksimovic, and Z. Popović, "A planar 4.5-GHz DC-DC power converter," IEEE Trans. Microw. Theory Techn., vol. 47, no. 8, pp. 1457-1460, Aug. 1999.

[20] M. N. Ruiz, R. Marante, and J. A. Garcia, "A class E synchronous rectifier based on an e-pHEMT device for wireless powering applications," in IEEE MTT-S Int. Microw. Symp. Dig., Montréal, QC, Canada, 2012, pp. 1-3.

[21] S. Imai, S. Tamaru, K. Fujimori, M. Sanagi, and S. Nogi, "Efficiency and harmonics generation in microwave to dc conversion circuits of half-wave and full-wave rectifier types," in IEEE MTT-S Int. Microw. Symp. Dig., 2011, pp. 15-18, Workshop ser. Innovative Wireless Power Transmission: Technol., Syst., Appl..

[22] H. Takhedmit, B. Merabet, L. Cirio, B. Allard, F. Costa, C. Vollaire, and O. Picon, "A 2.45-GHz low cost and efficient rectenna," in Proc. 4th Eur. Antennas Propag. Conf., Apr. 2010, pp. 1-5.

[23] R. Marante, M. N. Ruiz, L. Rizo, L. Cabria, and J. A. Garcia, "A UHF class E2 DC/DC converter using gan hemts," in IEEE MTT-S Int. Microw. Symp. Dig., Jun. 2012, pp. 1-3.

[24] M. Kazimierczuk and J. Jozwik, "Analysis and design of class E zerocurrent-switching rectifier," IEEE Trans. Circuits Syst., vol. 37, no. 8, pp. 1000-1009, Aug. 1990.

[25] F. Raab, "Class-E, Class-C, and Class-F power amplifiers based upon a finite number of harmonics," IEEE Trans. Microw. Theory Techn., vol. 49, no. 8, pp. 1462-1468, Aug. 2001.

[26] F. Raab, P. Asbeck, S. Cripps, P. Kenington, Z. Popovic, N. Pothecary, J. Sevic, and N. Sokal, "Power amplifiers and transmitters for RF and microwave," IEEE Trans. Microw. Theory Techn., vol. 50, no. 3, pp. 814-826, Mar. 2002.

[27] S. Kee, I. Aoki, A. Hajimiri, and D. Rutledge, "The class-E/F family of ZVS switching amplifiers," EEE Trans. Microw. Theory Techn., vol. 51, no. 6, pp. 1677-1690, Jun. 2003.

[28] M. Roberg and Z. Popović, "Analysis of high-efficiency power amplifiers with arbitrary output harmonic terminations," IEEE Trans. Microw. Theory Techn., vol. 59, no. 8, pp. 2037-2048, Aug. 2011.
[29] M. Roberg, E. Falkenstein, and Z. Popović, "High-efficiency harmonically-terminated rectifier for wireless powering applications," in IEEE MTT-S Int. Microw. Symp. Dig., Montréal, QC, Canada, 2012, pp. 1-3.

[30] M. Roberg, J. Hoversten, and Z. Popović, "GaN HEMT PA with over $84 \%$ power added efficiency," Electron. Lett., vol. 46, no. 23, pp. 1553-1554, Nov. 2010.

[31] "Swap-x402 data sheet," VTD, Brive, France, 2011. [Online]. Available: http://www.vtd-rf.com/pdf/productpresentation.pdf

[32] J. Verspecht, "Calibration of a measurement system for high frequency nonlinear devices," Ph.D. dissertation, Vrije Univ. Brussel (VUB), Brussels, Belgium, 1995.

[33] G. Callet, J. Faraj, O. Jardel, C. Charbonniaud, J.-C. Jacquet, T. Reveyrand, E. Morvan, S. Piotrowicz, J.-P. Teyssier, and R. Quéré, "A new nonlinear hemt model for AlGaN/GaN switch applications," Int. J. Microw. Wireless Technol. (Special Issue), vol. 2, no. 3-4, pp. 283-291, 2010.

[34] J. Faraj, G. Callet, O. Jardel, A. El-Rafei, F. De Groote, R. Quéré, and J. Teyssier, "Time domain large signal characterization of self-biasing phenomena in switch-mode AlGaN/GaN hemts," in 74th ARFTG Symp./IEEE MTT-S Int. Microw. Symp. Dig., Montréal, QC, Canada, 2012.

[35] M. Roberg, "Analysis and design of non-linear amplifiers for efficient microwave transmitters," Ph.D. dissertation, Univ. Colorado at Boulder, Boulder, CO, 2012.

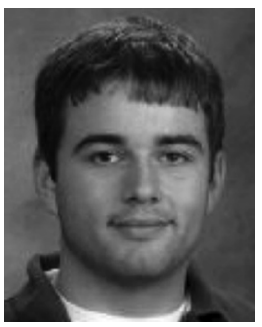

Michael Roberg (S'09) received the B.S.E.E. degree from Bucknell University, Lewisburg, PA, in 2003, the M.S.E.E. degree from the University of Pennsylvania, Philadelphia, in 2006, and the Ph.D. degree from the University of Colorado at Boulder in 2012.

From 2003 to 2009, he was an Engineer with Lockheed Martin-MS2, Moorestown, NJ, where he was involved with advanced phased-array radar systems. He is currently with the Defense Products and Foundry Services, TriQuint Semiconductor, Richardson, TX, where she is involved with wideband high-efficiency GaN MMIC PA design. His current research interests include high-efficiency microwave PA theory and design, microwave power rectifiers, monolithic microwave integrated circuit (MMIC) design, and high-efficiency radar and communication system transmitters.

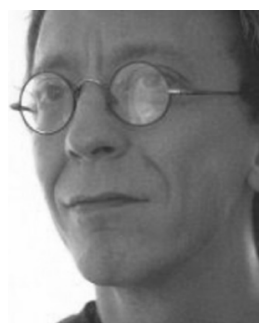

Tibault Reveyrand (M'07) received the Ph.D. degree from the University of Limoges, Limoges, France, in 2002. From 2002 to 2004, he was a Post-Doctoral Scientist with CNES (French Space Agency). In 2005, he became a CNRS Engineer with the XLIM Laboratory, University of Limoges, Limoges, France. His research interests include the characterization and modeling of RF and microwave nonlinear components and devices.

Dr. Reveyrand is a member of the IEEE MTT-11 "Microwave Measurements" Technical Committee. He was the recipient of the 2002 European GaAs Best Paper Award.

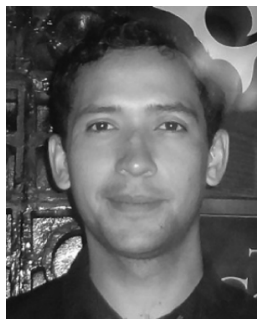

Ignacio Ramos (S'12) received the B.S. degree in electrical engineering from the University of Illinois at Chicago, in 2009, and is currently working toward the Ph.D. degree at the University of Colorado at Boulder.

From 2009 to 2011, he was with the Power and Electronic Systems Department, Raytheon IDS, Sudbury, MA. His research interests include high-efficiency microwave PAs, microwave dc/dc converters, radar systems, and wireless power transmission. 


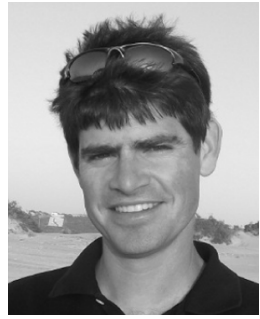

Erez Avigdor Falkenstein (S'07) was born in Haifa, Israel, in 1979. He received the "Handesaie" degree (associate degree) in electronics from the Amal Handesaim School Hadera, Hadera, Israel, in 1999, and the concurrent M.S./B.S. degrees in electrical engineering and $\mathrm{Ph} . \mathrm{D}$. degree from the University of Colorado at Boulder, in 2010 and 2012, respectively.

From 1999 to 2003, he was with the Israel Defense Force, as part of a technological unit. From 2004 to 2012, he was with the University of Colorado at Boulder. Since 2007, he has been involved with research as part of the Active Antenna Group. He is currently with Qualcomm Inc., Boulder, CO. His research concerns far-field wireless powering for low-power densities. His research interests include antenna design and characterization, modeling and measurement of nonlinear devices at microwave frequencies, and power management.

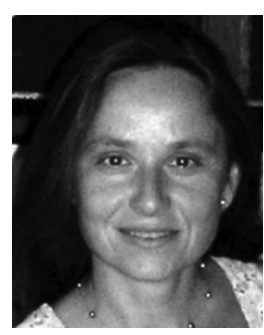

Zoya Popović (S'86-M'90-SM'99-F'02) received the Dipl.Ing. degree from the University of Belgrade, Belgrade, Serbia, Yugoslavia, in 1985, and the Ph.D. degree from the California Institute of Technology, Pasadena, in 1990. Since 1990, she has been with the University of Colorado at Boulder, where she is currently a Distinguished Professor and holds the Hudson Moore Jr. Chair with the Department of Electrical, Computer and Energy Engineering. In 2001, she was a Visiting Professor with the Technical University of Munich, Munich, Germany. Since 1991, she has graduated 44 Ph.D. students. Her research interests include high-efficiency, low-noise, and broadband microwave and millimeter-wave circuits, quasi-optical millimeter-wave techniques, active antenna arrays, and wireless powering for batteryless sensors. Prof. Popović was the recipient of the 1993 and 2006 Microwave Prizes presented by the IEEE Microwave Theory and Techniques Society (IEEE MTT-S) for the best journal papers and the 1996 URSI Issac Koga Gold Medal. In 1997, Eta Kappa $\mathrm{Nu}$ students chose her as a Professor of the Year. She was the recipient of a 2000 Humboldt Research Award for Senior U.S. Scientists of the German Alexander von Humboldt Stiftung. She was elected a Foreign Member of the Serbian Academy of Sciences and Arts in 2006. She was also the recipient of the 2001 Hewlett-Packard (HP)/American Society for Engineering Education (ASEE) Terman Medal for combined teaching and research excellence. 